\title{
Peripheral Intravenous Catheter (PIVC) Related Local Complications among Patients in KFCH-Jizan
}

\section{Makafi SA*and Marfega MACM}

Nursing Education and Staff Development Department, King Fahd Central Hospital-Jizan, Saudi Arabia

*Corresponding author: Shougi A Makafi, Nursing Education and Staff Development Department, King Fahd Central Hospital-Jizan, Saudi Arabia , Tel: +966568803213;E-mail: shougi.makafi@gmail.com

Received date: March 13, 2017; Accepted date: July 26, 2017; Published date: August 2, 2017

Copyright: @ 2017 Makafi SA, et al. This is an open-access article distributed under the terms of the Creative Commons Attribution License, which permits unrestricted use, distribution, and reproduction in any medium, provided the original author and source are credited.

\begin{abstract}
Background: Peripheral intravenous catheterization is a common invasive nursing procedure performed in healthcare institutions. It is a technique which involves insertion of a needle into the peripheral veins for various reasons. As one of the commonest nursing procedures being performed, it also brought significant risks to patients resulting to prolong hospital stay and delay in recovery period if not properly executed.
\end{abstract}

Objectives: The study primarily aims to determine the incidence rate of peripheral intravenous cannula related local complications in the hospital.

Design: The study is a descriptive type of research since it mainly deals with identifying the incident rate of phlebitis, infiltration, extravasation and hematoma and its underlying causes.

Settings: Study was conducted in one of the Ministry of Health hospitals in Jazan, Saudi Arabia.

Participants: All the patients admitted in the hospital during the data gathering period was included regardless of their demographic status. A total of 406 patients, with 458 peripheral cannula were assessed.

Methods: The study is quantitative type of research since it mainly sought to find out the number of phlebitis, infiltration, extravasation and hematoma cases in the above mentioned healthcare institution.

Result: The findings show that the rate of phlebitis, infiltration, extravasation and hematoma are $21,7,3.5,12$ percent respectively.

Conclusions: There were high incidence of intravenous cannula related complications in the hospital which needs to be reduced or completely eradicated in order to improve overall patient's health outcome.

Keywords: Cannulation; Complications; Extravasation; Hematoma; Infiltration; Intravenous; Nursing; Peripheral; Phlebitis; Venipuncture

\section{Introduction}

Peripheral intravenous catheterization (PIVC) is a common invasive procedure performed in hospitals. PIVC is a technique involves insertion of a needle into the peripheral veins for different reasons. It is used to save lives of patients by different means, however, in some cases became an additional risk. Risk includes local complications related PIVC such as phlebitis [1]. Phlebitis is the inflammation of the veins which may be due to mechanical, chemical, or infectious causes [2]. It can be associated with symptoms such as warmth, tenderness, redness, and/or swelling at the insertion site. Extravasation can be associated with milder symptoms of inflammation such as tenderness, erythema, redness, and warmth at the affected area or in severe symptoms such as tissue necrosis. In these cases inflammation can track further along the length of the vein resulting indurations and palpable venous cord. Damages due to extravasation can also extend to nerves, tendons, and joints [3]. Infiltration occurs when nonvesicant fluids or medication infiltrate into the subcutaneous tissue causing edema, skin blanching and coldness, and pain [4]. Hematoma formation occurs due to collection of blood from ruptured vessels which led to swelling at the affected area and cause pain [5].

The purpose of this study is to identify the incident rate of phlebitis, extravasation, infiltration and hematoma among patients in King Fahd Central Hospital (KFCH) Jazan. Furthermore is to find some of the risk factors associated with PIVC related complications.

\section{Methodology}

Study design is descriptive type since it focuses in determining the incidence of phlebitis, extravasation, infiltration, hematoma and identifying its risk factors related to its occurrence.

Simple statistical analysis was used in order to interpret data gathered, such as counting, frequency and percentage technique.

\section{Data collection and tool}

The data collection instrument was self-developed tool based on complications guidelines. Phlebitis clinical signs and symptoms 
include pain, redness, warm to touch, and swelling [2]. In order to differentiate infiltration versus extravasation, the researchers made an in-depth study of the current medications and intravenous fluids each patient is taking, as we rely on vesicant and non-vesicant solutions. Moreover, clinical manifestations of the patients were also considered. Infiltration signs and symptoms include swelling, cool to touch, and pain [4]. In the other hand, extravasation clinical signs and symptoms are pain, redness, swelling and in advance stages blisters maybe presented. Leakage is either reported by a patient or visually present in the dressing site. Hematoma usually developed due to improper technique in performing venipuncture. Reddish discoloration in and around the injection site signifies hematoma formation [5]. Visual assessment was used to identify this type of complication.

The tool also included different variables related to participants such as age and gender; staff nurses compliance to hospital policies and procedures in IVC documentation after insertion, site labeling, removal of the IVC or in the presence of any complications, dwelling time, reasons of insertion, number of IVCs inserted, dressing material used to secure the insertion site.

Two departments per day were assessed. For new IVC insertion or changing after the researcher visit a reporting form where given to the departments in order to notify the researchers to go and assess the newly inserted IVC and old IVC site. The reporting form includes the date and time of changing, patient's record number, department, and the reason for changing.

\section{Participants and ethical approval}

Purposive sampling method was used for this study. The participants were all patients with peripheral IV access and admitted in KFCH-JAZAN. The data collection included 406 patients and 458 cannula sites. It was conducted over one month period starting November 22, 2015 and finished on December 24, 2015.

Ethical approval obtained from the hospital Research Ethical Committee (REC). Verbal consent was obtained from conscious patients, guardians or immediate relative was contacted for subjects who were unable to provide consent. A courtesy call to floor supervisors and charge nurses was done to explain the study and to gain their permission to start the assessment.

\section{Results}

There were 406 patients with 458 cannula sites were inspected. Out of 406 participants 261 were male and 145 were female. PIVC was inserted for different reasons such as administration of fluids, IV medications, blood or blood products, blood extractions, radiological contrast solutions, and to keep veins open for any emergency situations. Cannula gauge 22 was noted in 163 patients, gauge 20 was with 147 patients, and largest gauge used was 16 which was seen in 3 patients. Four hundred twenty-five (93\%) cannulas were inserted in upper extremities such as hands or forearms. Three hundred fiftyseven patients were with one cannula, 46 patients with two cannula and 3 patients were with three cannula inserted. Fifty six percent (256 cannula sites) of IVC sites where labeled based on hospital policies while 44\% (202 cannula sites) either not labeled or incomplete. For documentation after cannulation, only $21 \%$ (96 sites) were documented completely, whereas $33 \%$ were over dated. Majority used gauze, elastic bandage, or non-adhesive paper tapes in securing PIVC, and use of transparent dressing accounts only for 142 sites (31\%).

\section{Discussion}

Nurse's scope of practice includes insertion of PIVC and is associated with risks and complications that might have an impact on patients' well-being. This study was undertaken to determine the incidence rates of (PIVC) related to local complications and to investigate various risk factors leading to phlebitis, extravasations, infiltration, and hematoma at $\mathrm{KFCH}$. The incidence of phlebitis, extravasation, infiltration, and hematoma was $21 \%, 3.5 \%, 7 \%$ and $12 \%$ respectively. Infusion Nurses Society (INS) recommend that the acceptable rate of phlebitis is $5 \%$ or less in any given population [6]. With regards to extravasation, infiltration, and hematoma there were no benchmarks documented regarding the acceptable rate. Nurse's goal should be at zero percentage. Therefore, adherence to hospital policies and guidelines in documentation, labeling, maintaining dwelling time and proper dressing is of paramount importance.

The rate of complications due to PIVC may also increase with several risk factors such as patients' age and sex. Increased age and having abnormal veins formation associated with increased risk of infection. However, in this study the researchers found out that there is no relation between the increased in age group and the incidents of phlebitis, infiltration, and hematoma. It was found that the higher rate for phlebitis belongs to the age group of 22-31years old was $4 \%$ from the total studied group. For the infiltration it was found the highest incidence in the age group of 12-21 years old which was $2.6 \%$. Hematoma cases occurs to the age group 32-41 years old which accounts for $1.4 \%$. Study of Boyd et al. supports the result of the study that extravasation incidence happens mostly to those 62-71 years old. On the other hand, studies of Osei-Tutu et al. and Kaur et al. upholds the results of the study which revealed that female are more likely to develop complications than male in terms of infiltration and hematoma which accounts for $5.8 \%$ and $4.59 \%$ respectively $[7,8]$. In contrast the result about the incidence of phlebitis and extravasation $(12.66 \%$ and $17.5 \%)$ were more likely to male than female.

Despite the evidence based guidelines by Royal College of Nursing (RCN) (2010) regarding the optimal time for changing the PIVC and post insertion documentation to be included in patients' records [9]. Presently, there is poor compliance in the nursing staff regarding documentations and optimal dwelling time to change PIVC. It was revealed that $79 \%$ of all PIVC inserted were either not documented or incomplete. Furthermore, $67 \%$ of PIVC inserted were over dated. Kaur et al stated that PIVC remaining in situ for greater than 72 hours were associated with a high rate of PIVC incidents [10]. It will also lead to increased hospitalization days of patients [11]. Therefore, RCN (2010) guidelines recommended that the cannula site must be assessed regularly through the use of transparent dressing, documentation and routine changing within 48-72 hours. Data gathered shows that only $31 \%$ were using transparent dressing and $69 \%$ were using either gauze, elastic bandage or none adhesive paper tapes, which made difficult to inspect the site regularly.

According to Morris and Heong Tay (2008) the higher rate of complication is associated with the increase number of IVC inserted in each patients [12]. This was different from the result found from the data gathered. The researchers found only $4 \%$ of the total participants has complications and with more than one cannula inserted. This study shows that phlebitis is associated more likely with IVC gauge 20 and 22 with $13 \%$ of total inspected PIVC sites. However, Nassaji and Ghibani (2007) claimed that the larger the diameter of the intravenous cannula the higher the chances of having phlebitis [13]. 
Citation: Makafi SA, Marfega MACM (2017) Peripheral Intravenous Catheter (PIVC) Related Local Complications among Patients in KFCHJizan. Adv Practice Nurs 2: 138. doi:10.4172/2573-0347.1000138

Page 3 of 3

\section{Conclusion}

Cannulation is a routine nursing procedure. This paper shows the rate of different PIVC local complications which previously not established. The rate of phlebitis is $21 \%$, infiltration $7 \%$ extravasation $3.5 \%$ and hematoma $12 \%$. The rise in complication rates among patients in $\mathrm{KFCH}$ need to be reduced for overall patient's outcome. This study can serve as a reference material for future researches related to PIVC complications as well as improving nursing care.

\section{Recommendations}

- With high rate of complications national and international IV cannulation standards and policies can be implemented in order to improve nursing work.

- Further studies to investigate different grades and causes of phlebitis in order to prevent it from developing.

- Further investigate nurses knowledge on IV cannulation practice to determine point of errors if present.

- National wide study to reveal where are we compared to international level and standard.

- Develop improvement projects to reduce PIVC related complications and improve patients' safety issues.

- Standardize IV cannulation protocols and policies to monitor and reduce cannula site complications.

- Further investigate on relation to cannula diameter inserted in the type of veins and the onset of PIVC related complication.

\section{References}

1. Chopra V, Montoya A, Joshi D, Becker C, Brant A, et al. (2015) Peripherally inserted central catheter use in skilled nursing facilities: A Pilot study. J Am Geriatr Soc 63: 1894-1899.
2. 2017 Nursing Practice. Phlebitis: treatment, care and prevention. Nursing Times 107.

3. Al-Benna S, O’Boyle C, Holley J (2013) Extravasation injuries in adults. ISRN Dermatol 2013; 856541.

4. Saini R, Agnihotri M, Gupta A, Walia I (2011) Epidemiology of infiltration and phlebitis. Nursing and Midwifery Research Journal 7: 22-33.

5. Buowari OY (2013) Complications of venepuncture. Advances in Bioscience and Biotechnology 4: 27656.

6. (2011) Infusion Nurses Society. Infusion nursing standards of practice 34.

7. Boyd S, Aggarwal I, Davey P, Logan M, Nathwani D (2011) Peripheral intravenous catheters: The road to quality improvement and safer patient care. J Hosp Infect 77: 37-41.

8. Osei-Tutu E, Tuoyire DA, Debrah S, Ayetey H (2015) Peripheral intravenous cannulation and phlebitis risk at Cape Coast teaching hospital. Postgraduate Medical Journal of Ghana 4: 11-18.

9. (2010) Royal College of Nursing. Standards for infusion therapy.

10. Kaur P, Thakur R, Kaur S, Bhalla A (2011) Assessment of risk factors of phlebitis amongst intravenous cannulated patients. Nursing and Midwifery Research Journal 7: 106-114.

11. Machado AF, Pedreira ML, Chaud MN (2008) Adverse events related to the use of peripheral intravenous catheters in children according to dressing regimens. Rev Lat Am Enfermagem 16: 362-367.

12. Morris W, Heong Tay M (2008) Strategies for preventing peripheral intravenous cannula infection. Br J Nurs 17: 14-21.

13. Nassaji ZM, Ghorbani M (2007) Peripheral intravenous catheter related phlebitis and related risk factors. Singapore Med J 48: 733-736. 